\title{
DIDÁCTICA Y DIDÁCTICA DE LA GEOGRAFÍA: BASES DE SU UNIDAD TEÓRICO-METODOLÓGICA
}

\author{
Pedro Álvarez Cruz ${ }^{1}$
}

Universidad de La Habana

Recibido 29/10/2019 Aceptado 09/06/2020

El presente artículo de reflexión teórica tiene como objetivo explicar las relaciones esenciales existentes entre la didáctica y la didáctica de la Geografía, así como su unidad teórico-metodológica. En su elaboración se utilizó una metodología de investigación documental; apoyada en diversas técnicas, tales como: lectura de fuentes documentales, recopilación de datos y presentación de la información. El artículo muestra las relaciones esenciales existentes entre la didáctica y las didácticas especiales; las cuales se expresan en los tres niveles siguientes: 1) general-particularsingular; 2) abstracto-concreto-generalización; y 3) teórico-metodológico-práctico. El análisis de estos niveles de relación revela, esencialmente, los fundamentos filosóficos, sociológicos, psicológicos, pedagógicos y didácticos; que constituyen bases de la unidad teórico-metodológica de la didáctica y las didácticas especiales.

$\exists$ This article of theoretical reflection aims to explain the essential relationships between the didactics and didactics of Geography, as well as its theoretical-methodological unit. In its elaboration, a documentary research methodology was used; supported by various techniques, such as: reading documentary sources, data collection and presentation of information. The article shows the essential relationships between didactics and special didactics; which expressed in the following three levels: 1) general-particular-singular; 2) abstract-concrete-generalization; and 3) theoretical-methodological-practical. The analysis of these levels of relationship reveals, essentially, the philosophical, epistemological, sociological, psychological, pedagogical and didactic foundations, which constitute the basis of the theoretical-methodological unit of didactics and special didactics.

DOI

https://doi.org/10.15366/didacticas2021.24.001

PALABRAS CLAVE

Didáctica; Didáctica especial; Didáctica de la Geografía. 


\section{INTRODUCCIÓN}

La didáctica es consubstancial a la formación de maestros y profesores para cualquiera de los niveles educacionales. Esta se manifiesta en estrecha relación con las didácticas especiales de las distintas disciplinas o asignaturas académicas. La didáctica y las didácticas especiales, asumidas como ciencias pedagógicas, se complementan una a otra desde postulados generales y particulares.

La didáctica, general por su contenido, tiene un carácter abstracto y generalizador que hace énfasis en los fundamentos teóricos de su objeto de estudio. Por su parte, las didácticas especiales, particular por su contenido, tienen un carácter concreto y singular que hace énfasis en los fundamentos metodológicos de su objeto de estudio. Estas características esenciales han devenido en interpretaciones que si bien reconocen la estrecha relación entre estas ciencias pedagógicas, por otra niegan su unidad teórico-metodológica al limitar su campo de acción a lo teórico o a lo metodológico. Lo que conlleva a la visión reduccionista del objeto de estudio de la didáctica y las didácticas especiales; así como a la segmentación de sus funciones teóricas y metodológicas.

La didáctica de la Geografía se ha encontrado en algunos momentos de su evolución en esa disquisición reduccionista al considerarse como método o concebida como metodología de enseñanza. Soslayando así, su función teórica y, por ende, metodológica manifiestas en unidad dialéctica. La presente reflexión tiene como objetivo explicar las relaciones esenciales existentes entre la didáctica y la didáctica de la Geografía, así como su unidad teórico-metodológica.

\section{DIDÁCTICA Y DIDÁCTICAS ESPECIALES: RELACIONES ESENCIALES EXISTENTES}

La didáctica y las didácticas especiales surgen integradas. Esto se aprecia en la obra de sus fundadores Wolfgang Ratke (Ratichus, 1571-1635) y Jan Amos Komenský (Comenio, 1592-1760). A Ratichus corresponde el mérito de emplear por primera vez el término didáctica (arte de enseñar) en su libro Principales aforismos didácticos (1629). Fue Comenio quien al valorar la importancia de la implantación de la didáctica, hizo evidente las relaciones entre el "arte de enseñar y aprender" y el "método de enseñar y aprender" como un todo viabilizado por los procedimientos de este último particular.

Ya desde los tiempos de Comenio quedó definida la función teórica, metodológica y práctica de la didáctica y, en particular, la función del "método de enseñar y aprender", al que trató diferenciado en: métodos de las ciencias en particular, de las artes, de las lenguas, de las costumbres y de inculcar la piedad. Preconizando así, tal vez, el surgimiento de las didácticas o metódicas especiales.

Así se comportó la evolución de la didáctica durante los siglos XVII, XVIII y XIX hasta que en la primera mitad del siglo XX fueron surgiendo las didácticas o metódicas especiales de las distintas disciplinas o asignaturas académicas. Diferenciándose de este modo el 
objeto de estudio de la didáctica y las didácticas especiales; el cual se expresa en los tres niveles de relaciones siguientes: 1) general-particular-singular; 2) abstracto-concreto-generalización; y 3) teórico-metodológico-práctico.

Según Klingberg (1978) en la relación existente entre la didáctica y las didácticas especiales pueden distinguirse tres etapas de desarrollo:

- Primera etapa: Vínculo muy estrecho entre la didáctica y las didácticas especiales. Las didácticas especiales o metodologías consistían solamente en la aplicación de los principios generales de la didáctica.

- Segunda etapa: Consolidación de las didácticas especiales como disciplinas autónomas de la ciencia pedagógica. Esta autonomía se produce en dos direcciones: 1) autonomía con respecto a la didáctica y 2) autonomía de cada una de las didácticas especiales entre sí. En esta fase existe ciertamente el peligro de un desarrollo separado de cada teoría de la enseñanza, el peligro del aislamiento y la desatención de los fundamentos pedagógico-didácticos comunes a todos los problemas teóricos de la enseñanza y el aprendizaje.

- Tercera etapa [a futuro]: Se restablecerá la unidad entre la didáctica y las didácticas especiales en un plano más elevado. Esta será una unidad en la que cada didáctica especial habrá desarrollado su perfil lógico-científico peculiar, pero al mismo tiempo estará vinculada más estrechamente que antes con todas las otras didácticas especiales y con la didáctica mediante rasgos comunes, conocimientos generales, tareas, etc. Se impondrá cada vez más la necesidad de colocar en el centro del trabajo lo común de las distintas disciplinas o asignaturas académicas para la formación integral del ser humano.

Las etapas descritas por Klingberg (1978) son evidencia de una clara unidad teóricometodológica entre la didáctica y las didácticas especiales, así como a lo interno de cada una de estas. La didáctica especial aplica las teorías y metodologías generales de la didáctica, que utiliza los resultados de las didácticas especiales para su propio trabajo de generalización. Esta relación se consolidará por la tendencia a la necesaria integración entre las ciencias pedagógicas.

\section{EVOLUCIÓN Y DESARROLLO DE LA DIDÁCTICA DE LA GEOGRAFÍA EN CUBA: BOSQUEJO HISTÓRICO DE SU RELACIÓN CON LA DIDÁCTICA Y OTRAS CIENCIAS}

La formación docente en Geografía no existía antes del año 1964. La Geografía, como asignatura, era enseñada en las escuelas primarias (primero a sexto grados) y las escuelas primarias superior (séptimo a octavo grados) por maestros formados en las escuelas normales (1916); y en las escuelas secundarias (institutos de segunda enseñanza y escuelas profesionales) por profesores formados en la Escuela de Pedagogía de la Universidad de 
La Habana (1900-1959), así como por geógrafos especializados en la Facultad de Filosofía y Letras de dicha Universidad, sin ningún estudio de pedagogía o didáctica.

El surgimiento de la didáctica de la Geografía, como disciplina científico-académica, estuvo ligado a la creación, en el año 1964, de los planes de estudio para la formación docente en Geografía, en los institutos pedagógicos fundados el propio año y adscriptos a las tres universidades existentes en aquel momento: Universidad de La Habana (1728), Universidad Central de Las Villas (1952) y Universidad de Oriente (1947). Se considera que “(...) sus antecedentes históricos los encontramos en la Metodología General, cursada en las escuelas normales y en la Didáctica de las Escuelas Secundarias; disciplina que aparecía en el currículo de la carrera de Pedagogía". (Ministerio de Educación, Cuba, 1975, p.1)

Por tanto, en el análisis histórico de la didáctica de la Geografía se sigue su tránsito por los diferentes planes de estudio de la formación docente en Geografía desde el propio año 1964 como se aprecia en Alvarez, Pérez y Recio (2015).

Todo lo antes expresado permite establecer los cuatro períodos evolutivos de la didáctica de la Geografía siguientes (Cuadro.1):

\begin{tabular}{|c|c|c|}
\hline Plan de estudio & Titulación & Períodos evolutivos \\
\hline 1964-1971 & Profesor de Geografía Historia & \multirow{2}{*}{ Iniciación (1964-1976) } \\
\hline 1972-1976 & $\begin{array}{l}\text { Profesor de Geografía para la Educación } \\
\text { General Media }\end{array}$ & \\
\hline $\begin{array}{l}\text { 1977-1989 } \\
\text { Plan A 1977-1981 } \\
\text { Plan B 1982-1989 }\end{array}$ & $\begin{array}{l}\text { Licenciado en Educación en la Especialidad de } \\
\text { Geografía }\end{array}$ & \multirow{2}{*}{$\begin{array}{l}\text { Consolidación (1977- } \\
\text { 2002) }\end{array}$} \\
\hline Plan C 1990-2002 & $\begin{array}{l}\text { Licenciado en Educación en la Especialidad de } \\
\text { Geografía }\end{array}$ & \\
\hline $\begin{array}{l}\text { Plan C (modificado) } \\
\text { 2003-2009 }\end{array}$ & $\begin{array}{l}\text { - Licenciado en Educación, Profesor General } \\
\text { Integral de Secundaria Básica } \\
\text { - Licenciado en Educación, Profesor de Ciencias } \\
\text { Naturales }\end{array}$ & $\begin{array}{l}\text { Postergación (2003- } \\
\text { 2009) }\end{array}$ \\
\hline Plan D 2010-2016 & Licenciado en Educación, Biología-Geografía & \multirow{2}{*}{$\begin{array}{l}\text { Revalorización (2010-ac- } \\
\text { tualidad) }\end{array}$} \\
\hline Plan E 2016-actualidad & Licenciado en Educación Geografía & \\
\hline
\end{tabular}

Cuadro 1. Períodos evolutivos de la didáctica de la Geografía en Cuba. Fuente: Elaboración propia.

En el período de iniciación (1964-1976), como su denominación indica, surgió la didáctica de la Geografía como disciplina científico-académica independiente de la formación docente en Geografía. Es importante destacar que los fundamentos de la 
disciplina se erigieron desde los postulados de la Escuela Nueva (Pedagogía Progresista) introducidos por varios pedagogos entre los que destacó el puertorriqueño, con residencia en Cuba, Alfredo Miguel Aguayo Sánchez (1866-1948).

En este período inicial va influir, de modo trascendental, la pedagoga cubana Graciela Barraqué Nicolau (1925-2001), quien se desempeñó como profesora de Ciencias Sociales y directora del Colegio Academia Barraqué (1957-1960), así como Jefa de Departamento de Geografía (1964-1971) en el Instituto Pedagógico Enrique José Varona de La Habana. Barraqué fue autora de los programas de estudio de didáctica de la Geografía, así como de numerosos folletos mimeografiados que sirvieron, dada la escasez de libros, como textos básicos para su enseñanza durante este período y los posteriores.

La didáctica de la Geografía se caracterizó, en su transmisión académica, por una lógica deductiva. Esta lógica quedó respaldada con los contenidos pedagógicos, didácticos y geográficos estudiados en años precedentes. Sus contenidos se orientaron a la sistematización del trabajo docente, al integrar las exigencias de la ciencia geográfica con los principios didácticos específicos inherentes a la enseñanza de esta disciplina.

A finales de los años '60 se hizo notable la influencia del pensamiento científico y pedagógico de los países socialistas de la Europa del Este (EE) y la Unión de Repúblicas Socialistas Soviéticas (URSS), que extendieron su apoyo al país más allá de la ayuda económica, por lo que se incrementaron los cursos de superación científica y metodológica de asesores, fundamentalmente, de procedencia soviética. Este hecho, indudablemente, condicionó la orientación hacia las características de la pedagogía y la didáctica de dichos países.

Una obra clave en la interpretación de estas cuestiones fue La lección de Geografía en la escuela (1966) del pedagogo soviético N. N. Studensov. Este libro escrito para los profesores de Geografía de la URSS sirvió como material de consulta, por su carácter general y aplicado, a los profesores de Geografía cubanos. Una cuestión interesante que se trató por el autor fue la relación entre la didáctica y la didáctica de la Geografía:

"El trabajo metodológico de muchos años con los profesores ha convencido al autor de este libro de que muchas deficiencias en las lecciones de Geografía son debidas a la incapacidad por parte de los maestros de aplicar las leyes y reglas de la didáctica a la enseñanza de la geografía (...)".

“(...) resulta que los postulados didácticos generales se exponen en su aspecto más general sin concretarlos en lo concerniente a la enseñanza de las diversas asignaturas, (...), mientras las cuestiones de metodología de la geografía se enseñan separadas de la didáctica general (...) la tendencia a excluir la metodología de la esfera de acción de la didáctica general se hace muy polémica e inútil, ejerciendo una influencia negativa sobre el trabajo educativo de los profesores de Geografía (...)”. (p.3) 
Más adelante señaló:

“(...) La ausencia hasta los últimos tiempos de un trabajo especial acerca de la lección de Geografía, nos permite esperar que este manual, aunque no pretende llenar la laguna indicada, de todas maneras pueda ser útil al profesor de Geografía, fijando su atención en la lección como un acto didáctico único en que se unen la didáctica general y la metodología de la asignatura”. (p.5)

Estos hechos señalado por Studensov, soslayados antes en el quehacer didáctico, iniciaron una reflexión teórica sobre las relaciones esenciales existentes entre la didáctica y la didáctica de la Geografía, así como la relación de estas con otras ciencias que corroboraría en los años posteriores la unidad teórico-metodológica entre éstas.

En el período de consolidación (1977-2002) la didáctica de la Geografía cambió su denominación por Metodología de la enseñanza de la Geografía. Este criterio emanó del sistema clasificatorio de las ciencias pedagógicas en los países socialistas de la EE y, sobre todo, en la URSS.

La didáctica de la Geografía, en este período, mantuvo en su transmisión académica una lógica deductiva respaldada en los contenidos pedagógicos, didácticos y geográficos estudiados en años precedentes. Sus contenidos se orientaron a la sistematización del trabajo docente, al integrar las exigencias de la ciencia geográfica con los principios didácticos específicos inherentes a la enseñanza de esta disciplina.

En este período los contenidos didácticos de la Geografía se reflejaron en la obra Metodología de la enseñanza de la Geografía $(1978,1991)$ confeccionada por la profesora Graciela Barraqué Nicolau. Este libro sirvió de texto básico hasta el año 2002. En éste, su autora sistematizó de modo elocuente los resultados más avanzados en la materia y consultados en autores, tales como: Brouillette, et al. (1966), Studensov (1966), Tomaschewky (1974), Darinsky (1975), Danilov y Skatkin (1978), Klingberg (1978), Pancheshnikova, et al. (1983), Talízina (1985), entre otros.

Barraqué $(1978,1991)$ definió la metodología de la enseñanza de la Geografía como:

“(...) un componente del sistema de la ciencia pedagógica, y como ciencia pedagógica particular, constituye la teoría de la enseñanza y de la educación, que se manifiesta a través del proceso de aprendizaje geográfico, es decir, en la actividad docente teórico-práctica de las asignaturas geográficas”.

“(...) La metodología de la enseñanza de la Geografía está vinculada, directamente, con otras ciencias afines. Se encuentra estrechamente relacionada con la didáctica general y la teoría de la educación, pues utiliza los conceptos básicos de ambas ciencias en su campo específico de estudio, y a la vez, aporta los resultados de sus experiencias e investigaciones con las otras metodologías de las distintas enseñanzas, pues a pesar de las diferencias sustanciales que existen entre ellas, posee un centro de gravedad: los principios generales de la educación..., y con- 
tribuye conscientemente a través de las asignaturas de sus especialidades, a la formación del hombre nuevo y a la cientificidad del trabajo docente-educativo". (p. IX)

También expresó que:

"La metodología de la enseñanza de la Geografía es la piedra angular en la formación de todo profesor de Geografía, ya que constituye la fundamentación teórico-práctica del quehacer docente".

“(...) la metodología de la enseñanza de la Geografía, es la ciencia que garantiza el cumplimiento del perfil ocupacional del profesor, y su estudio precisa y estimula la vocación profesional. Ofrece los contenidos y la orientación imprescindibles para el dominio de las regularidades y principios metodológicos en el tratamiento de las asignaturas geográficas”. (p. XIII)

Barraqué, al asumir la metodología de la enseñanza de la Geografía como ciencia pedagógica particular, reveló su unidad teórico-metodológica devenida de las relaciones esenciales existentes entre ésta con la didáctica y las demás didácticas especiales; así como con el sistema de ciencias pedagógicas.

Barraqué estructuró su obra $(1978,1991)$ logrando una armonía entre lo teórico (didáctica y teoría de la educación) y lo metodológico (métodos de enseñanza de la Geografía), así como su implicación en la práctica docente sin caer en posiciones preceptivas. De los 13 capítulos escritos, seis (46,2\%) tratan cuestiones relacionadas con el método, y esto, desde una visión integradora entre los postulados emanados de la didáctica, y otras ciencias pedagógicas, con las particularidades del contenido geográfico en cada nivel educacional en correspondencia con la edad y características psicológicas de los alumnos.

Es importante destacar que a partir del año 1995 se realizaron un grupo de precisiones curriculares en las asignaturas geográficas, que derivaron en un reajuste curricular de la Geografía en los diferentes niveles educacionales; los que se generalizaron en el curso escolar 2001-2002. Recordar que años antes había caído el campo socialista en la EE y la URSS; cuestión que influyó en todo el quehacer político, económico y social de Cuba.

Todo esto trajo consigo un replanteamiento en la didáctica de la Geografía, que sin perder los logros alcanzados se actualizaría en correspondencia con la influencia de otras tendencias pedagógicas y didácticas propias de la tradición pedagógica cubana y mundial. Surgen así dos obras: Enrutando la Geografía Escolar (1999) escrita por un colectivo de autores bajo la conducción de Pedro Ángel Hernández Herrera; y Selección de temas de Didáctica de la Geografía (2002) escrita por un colectivo de autores bajo la dirección de Celina Esther Pérez Álvarez. 
En ambos libros se ratificó a la didáctica y a la didáctica de la Geografía como ciencias pedagógicas, así como la unidad teórico-metodológica entre ambas. Resultan interesantes las aclaraciones que sobre el uso indistinto de didáctica de la Geografía y metodología de la enseñanza de la Geografía se hicieran en períodos anteriores:

"Las tareas de esta ciencia [metodología de la enseñanza de la Geografía] descrita por dicha autora [Pancheshnikova (1983)] declaran su función teórica en justa concordancia con su esencia filosófica, que expresa que la metodología no es solo conjunto de procedimientos para la acción práctica, sino que cuenta con su aparato teórico de fundamentación epistemológica”.

"Para mayor exactitud en lo expuesto, se esclarece que Pancheshnikova y Barraqué, emplean el término metodología de la enseñanza de la Geografía (...) con la misma significación del de Didáctica de la Geografía, pues en ningún momento limitan su objeto a la prescripción y la normativa, que tampoco son las únicas vertientes de la metodología, como aparece en algunas fuentes".

"El empleo indistinto de estas denominaciones, didácticas especiales y metodología de las diferentes disciplinas académicas, puede no tener trascendencia, sin embargo, cuando se acepta a la metodología, solo como norma, guía o instrumento, es decir con un carácter únicamente indicativo, prescriptivo y normativo, la tiene; si queda clara la unidad dialéctica entre lo teórico y lo metodológico, no hay conflicto”.

"Los autores comparten el criterio de los que emplean Didáctica especial; porque consideran que el de Metodología, puede prestarse más a restringir el objeto y el campo de acción de la primera o bien, que se trata de dos disciplinas, que en su vínculo con la Didáctica general, expresan la relación entre lo general-lo particular-lo singular”. (Pérez y Recio, 2002, p.17)

Entre los años 2003 y 2009, la didáctica de la Geografía, entra en un período de postergación. En las nuevas concepciones de formación docente las relaciones entre la didáctica y las didácticas especiales sufren seria ruptura por la reducción de estas últimas a los contenidos y métodos de enseñanza de las asignaturas en el contexto escolar. La didáctica de la Geografía se diseñó de modo preceptivo y en correspondencia con los programas de Geografía de la enseñanza primaria, secundaria básica y preuniversitaria.

En este período se manifestó una carencia de los estudios sobre la didáctica de la Geografía. Las asignaturas geográficas, en el ámbito escolar, fueron guiadas por las exigencias básicas siguientes:

La función rectora de la asignatura en el trabajo de educación ambiental, a partir de lo cual garantizará el logro de los objetivos formativos orientados hacia este fin en el grado.

La derivación gradual de los objetivos generales en las unidades y clases para propiciar que los alumnos expresen su antiimperialismo y rechacen las políticas yanquis de agresión y bloqueo contra Cuba, mediante el análisis de datos, tablas, gráficas, documentos históricos, dis- 
cursos de dirigentes (...), para arribar a conclusiones acerca de la posición firme del pueblo cubano de mantener su independencia, soberanía y luchar por mantener las conquistas alcanzadas.

El trabajo con mapas en diferentes niveles: reproducción o reconstrucción de significados, interpretación y aplicación.

La ubicación espacial de hechos históricos, que han ocurrido y ocurren en el mundo y en particular en Cuba.

La integración entre los componentes de la naturaleza y de la sociedad, objeto de estudio de la ciencia geográfica, al realizar el estudio de objetos, procesos y fenómenos que ocurren a escala planetaria, regional, nacional y local.

La utilización de algoritmos de trabajo para el estudio de paisajes, países y regiones, seleccionadas. (Hernández, et al., 2002, pp.33-34)

No obstante, hay que advertir que el ordenamiento curricular de la Geografía escolar, en el período 2001-2019, presentó cierta inestabilidad condicionada por modificaciones y reajustes continuos en los diferentes grados escolares. Estas han estado determinadas, fundamentalmente, por los cambios llevados a cabo, como se ha explicado anteriormente, en el modelo de formación de profesores de Geografía entre los años 2003 y 2009. Ese zigzagueo curricular, coyuntural, si bien tuvo en cuenta la situación socioeconómica del país, por otra, no exploró lo suficiente la situación epistemológica de la ciencia geográfica y su consideración como fuente curricular. Tampoco se analizó con profundidad las relaciones esenciales existentes entre los fundamentos epistemológicos y didácticos. La didáctica de la Geografía centró su análisis, en lo académico y lo investigativo, en el logro de las exigencias básicas antes enunciadas.

Las problemáticas expuestas, en el período anterior, condujeron a un replanteamiento en la concepción de la formación docente en Geografía y, por ende, se diseñó un nuevo modelo curricular, en el que la didáctica de la Geografía entró en un período de revalorización (2010-actualidad). Retornó a la formación docente en Geografía como disciplina científico-académica con una lógica inductiva en su transmisión académica.

En este período, la didáctica de la Geografía se constituye como núcleo teórico-metodológico fundamental en el proceso de formación integral del profesor de Geografía; dado que lo capacita para la dirección del proceso de enseñanza y aprendizaje geográfico; como esencia del objeto de trabajo de la profesión. Su contenido favorece el desarrollo teórico-práctico, en el dominio de una parte importante, de las habilidades profesionales pedagógicas. Este se selecciona en estrecha relación con el contenido de las disciplinas geográficas y, en particular, con los de las disciplinas pedagógicas.

Estos fundamentos se defienden en la obra Didáctica de la Geografía (2018) escrita por un colectivo de autores bajo la conducción de Celina Esther Pérez Álvarez. Este libro se estructuró a partir de los componentes fundamentales (profesor-alumno-grupo-problema-objetivo-contenido-método-medio-forma organizativa-evaluación) del proceso ense- 
ñanza y aprendizaje en los tres niveles de relaciones siguientes: 1) general-particular-singular; 2) abstracto-concreto-generalización; y 3) teórico-metodológico-práctico. Una peculiaridad de esta obra es la reflexión sobre la necesidad una perspectiva teórica que oriente la modelación didáctica de la Geografía, dado la existencia de múltiples enfoques de la ciencia geográfica que se debate, tal vez, más que los de ninguna otra ciencia y que influyen en el diseño, desarrollo y evaluación del proceso de enseñanza y aprendizaje de la Geografía.

\section{BASES DE LA UNIDAD TEÓRICO-METODOLÓGICA DE LA DIDÁCTICA Y LA DIDÁCTICA DE LA GEOGRAFÍA}

Las bases de la unidad teórico-metodológica de la didáctica y la didáctica de la Geografía se definen en las relaciones que se establece con otras ciencias, que se constituyen como sus fuentes y fundamentos teórico-metodológicos para su aplicación práctica.

Didáctica-didáctica de la Geografía-filosofía. No hay ciencia que pueda existir sin la filosofía, sin una base metodológica. La didáctica y la didáctica de la Geografía no se reducen a la filosofía, tienen su objeto y sus tareas, pero tampoco pueden realizarse sin ésta. Al determinarse los objetivos de enseñanza y aprendizaje, la didáctica y la didáctica de la Geografía parten de una determinada concepción filosófica del mundo, así como de los fundamentos gnoseológicos (teoría del conocimiento) y epistemológicos (teoría del conocimiento de la ciencia).

En el estudio de los fundamentos gnoseológicos se determinan las fuentes, formas, métodos y procedimientos para la transmisión (profesor) y apropiación de conocimientos (alumno) necesarios para la vida en sociedad. En esta interacción (transmisión/enseñanza-asimilación/aprendizaje) se manifiesta la tarea principal de la didáctica: la asimilación y desarrollo cognitivo del alumno, lo que lo prepara desde la actividad de estudio para la actividad del trabajo y la vida en sociedad a partir de la apropiación de la cultura que le ha antecedido.

En el quehacer didáctico de la Geografía, en Cuba, en los últimos 55 años la formación de conceptos y habilidades ha estado orientada por la teoría del conocimiento materialista dialéctica. Estableciéndose los procedimientos para la formación conceptual siguientes: comparación, análisis, síntesis, abstracción y generalización; los cuales derivaron como requisitos didácticos para la formación de conceptos en la enseñanza de la Geografía (Ver Figura 1 y cuadro 2).

Studensov (1966) consideró que:

“(...) los conocimientos geográficos constan de: 1) las imágenes, 2) las nociones, y 3) los juicios; con la ayuda de los cuales se unen y se sistematizan las nociones. Para que los conocimientos adquiridos sean sólidos y para qué tengan significado práctico, se consolidan en los 

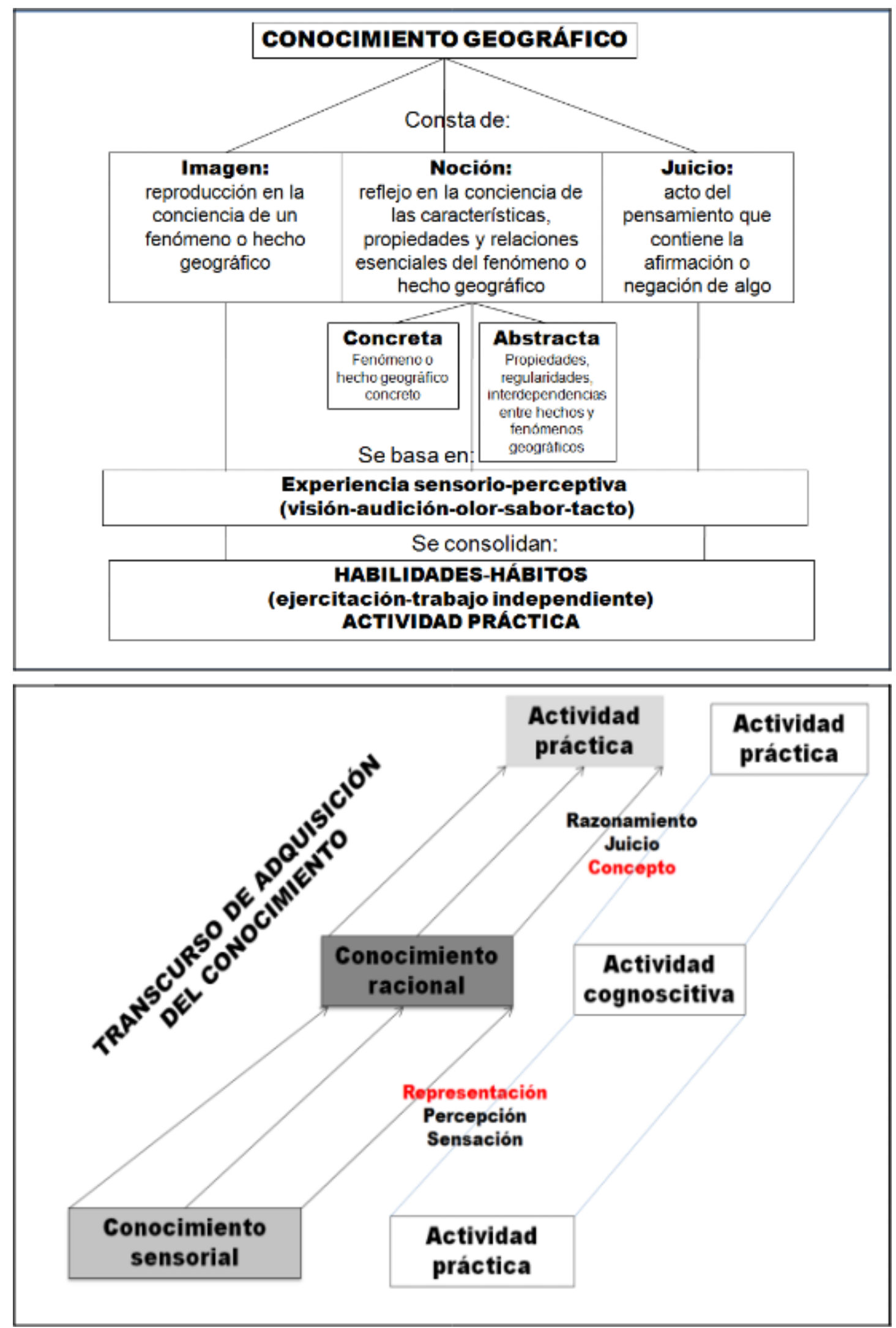

Fig.1. Aplicación de la teoría del conocimiento a la enseñanza de la Geografía. Fuente: Elaboración a partir de Studensov (1966) y Barraqué (1991). 
hábitos y las habilidades, los cuales se crean a través de los ejercicios, que contribuyen en muchos casos a una profundización o un esclarecimiento más completo de las nociones”. (p. 7)

Por su parte Barraqué $(1978,1991)$ asumió las representaciones y los conceptos como núcleos de los conocimientos geográficos; en los que su solidez y significación dependen de sus aplicaciones prácticas mediante un sistema de actividades vinculadas con la formación de habilidades. Además de eso, mostró la formación de conceptos geográficos por las dos vías siguientes:

\begin{tabular}{|c|c|c|}
\hline \multirow{2}{*}{ Pasos } & \multicolumn{2}{|c|}{ Razonamiento } \\
\hline & Inductivo & Deductivo \\
\hline 1 & $\begin{array}{l}\text { Observación del objeto, fenómeno o proceso } \\
\text { geográfico, su interpretación de lo observado por } \\
\text { el alumno, la descripción de la interpretación ob- } \\
\text { tenida, la integración de las características exter- } \\
\text { na de lo observado en una imagen mental sensi- } \\
\text { ble. }\end{array}$ & $\begin{array}{l}\text { Formulación de la definición del concepto } \\
\text { geográfico por el profesor o lectura de la } \\
\text { definición, por los alumnos, en el libro de } \\
\text { texto. }\end{array}$ \\
\hline 2 & $\begin{array}{l}\text { Representación, imagen de la memoria que re- } \\
\text { fleja lo percibido, es decir, sin encontrarse, pre- } \\
\text { sente el objeto o fenómeno geográfico. En este } \\
\text { paso entra en juego la imaginación. }\end{array}$ & $\begin{array}{l}\text { Análisis de la definición y su vinculación } \\
\text { con contenidos geográficos previamente } \\
\text { asimilados por los alumnos para la obten- } \\
\text { ción de una síntesis nueva. }\end{array}$ \\
\hline 3 & $\begin{array}{l}\text { Concepto, análisis de la representación obtenida } \\
\text { para extraer las características esenciales para } \\
\text { desechar lo accidental e integrar una síntesis que } \\
\text { precise las características intrínsecas de lo ob- } \\
\text { servado, que se refleje en el concepto mediante } \\
\text { la abstracción, y se generaliza a través de la } \\
\text { comparación de ejemplos de la realidad o de sus } \\
\text { ilustraciones. }\end{array}$ & $\begin{array}{l}\text { Generalización de la definición conceptual } \\
\text { sobre la base de la observación y la com- } \\
\text { paración de objetos, fenómenos o proce- } \\
\text { sos similares a lo definido, en la realidad o } \\
\text { mediante ejemplificaciones e ilustracio- } \\
\text { nes. }\end{array}$ \\
\hline 4 & $\begin{array}{l}\text { Juicio, definición por los alumnos del concepto y } \\
\text { precisión del límite de su alcance, características } \\
\text { suficientes y necesarias, por el profesor. }\end{array}$ & $\begin{array}{l}\text { Imagen mental (concreto-pensado) de lo } \\
\text { definido conceptualmente y precisión de } \\
\text { sus componentes esenciales mediante la } \\
\text { abstracción correspondiente. }\end{array}$ \\
\hline 5 & $\begin{array}{l}\text { Aplicación del concepto aprendido en las activi- } \\
\text { dades y tareas escolares. }\end{array}$ & $\begin{array}{l}\text { Aplicación del concepto aprendido en las } \\
\text { actividades y tareas escolares. }\end{array}$ \\
\hline
\end{tabular}

Cuadro 2. Vías para la formación de conceptos geográficos. Fuente: Elaboración a partir de Barraqué (1991)

Los fundamentos gnoseológicos, como se aprecia en los ejemplos anteriores, se hallan estrechamente relacionados con la psicología y, en particular, con la piscología evolutiva en su análisis del desarrollo del aprendizaje. 
En las relaciones entre la didáctica, la didáctica de la Geografía y la epistemología de la ciencia a enseñar y aprender, en este caso, de la ciencia geográfica. Se identifica, selecciona, clasifica, ordena el contenido (conocimientos, habilidades, hábitos, experiencia de la actividad creadora y normas de relación con el mundo) de la disciplina o asignatura académica a enseñar y aprender. Esto exige otro nivel de relación expresado con la teoría curricular, que tiene en cuenta fundamentalmente las fuentes filosóficas, sociológicas, psicológicas, pedagógicas y didácticas. La epistemología contiene, entre otros aspectos, los fundamentos de los tipos de conocimiento científico y el grado con el que cada uno resulta verdadero; así como la relación entre sujeto cognoscente-objeto cognoscible.

En el decurso de la Geografía y de su enseñanza, el proceso de comprensión de los fenómenos, procesos y hechos que se manifiestan en el espacio geográfico, como categoría esencial de esta ciencia, se ha revelado desde diferentes perspectivas teóricas (enfoques), que se sustentan desde diversas posiciones teóricas, por una comunidad científica, en un contexto espacio-temporal dado.

En la historia de la Geografía y de su enseñanza, el surgimiento de un nuevo enfoque no ha implicado la desaparición de los anteriores, que pueden perder o no su vigencia en el quehacer científico o educativo. Eso justifica una pluralidad creciente de posiciones teóricas y metodológicas para el estudio de la misma categoría: el espacio geográfico; el cual ha recibido un sinnúmero de denominaciones, lo que hace impreciso y polémico el objeto de estudio de la Geografía.

Esos enfoques geográficos se proyectan en el proceso de enseñanza y aprendizaje de la Geografía sirviéndole de fuente epistemológica; lo que es importante analizar por alumnos, profesores e investigadores, dado que:

\footnotetext{
“(...) estamos insertos en una realidad cambiante y compleja; la ciencia avanza no solo en conocimiento sino en producciones, metodologías y técnicas, hay múltiples miradas para interpretar la realidad y de acuerdo con las convicciones es la selección; los paradigmas dominantes ofrecen líneas para percibir y explicar o comprender los hechos, existe una producción conceptual profusa: surgen nuevos y variados conceptos; facilitan elaborar conocimiento para y con los alumnos; orientan las vías para abordar problemas específicos e investigar con los marcos conceptuales, las técnicas y los métodos propios de cada perspectiva y, en el caso particular de la enseñanza, orienta el tratamiento de los contenidos curriculares a desarrollar en cada uno de los niveles educativos".(Santarelli y Campos, 2008, s/p).
}

La enseñanza de la Geografía no puede ni debe existir al margen de dicha pluralidad de enfoques, sino que, su modelación didáctica debe sustentarse en esas bases epistemológicas, entrelazadas armónicamente a la realidad socioeconómica existente; manteniendo como premisa que todo lo que se haga se vea reflejado en la formación integral de las diferentes generaciones. 
Didáctica-didáctica de la Geografía-cibernética. La cibernética es la "ciencia que trata de los rasgos generales de los procesos y sistemas de dirección en los dispositivos técnicos, en los organismos vivos y en las organizaciones humanas". (Rosental y Iudin, 1973, p.64)

El proceso de enseñanza y aprendizaje se expresa en la interacción (comunicación) del profesor con el grupo y cada alumno. Estas relaciones son esenciales y marcadas por la actividad de estudio, en la que el profesor enseña y el alumno aprende. Addine (2004) señaló que en el proceso de enseñanza y aprendizaje se manifiestan las interrelaciones siguientes:

“(...) primero, lo humano, constituido por el maestro, sus estudiantes, el grupo en interacción constante y fecunda; segundo, lo cultural, formado por los objetivos, contenidos, método-formas de organización, evaluación. Será siempre grave distorsión de la perspectiva didáctica el atribuir importancia o énfasis exagerados al contenido o al método, como si fueran los únicos o decisivos de la situación; serán valiosos solo en cuanto contribuyan a desarrollar y formas la personalidad de los estudiantes". (p. 5)

Esto permite comprender al proceso de enseñanza y aprendizaje como una organización humana, en la que se establecen estrechas relaciones sistémicas de subordinación y coordinación entre cada uno de sus componentes mediados por los componentes culturales. Desde este punto de vista, la didáctica de la Geografía redefine su objeto de estudio como la dirección del proceso de enseñanza y aprendizaje geográfico. Y, por tanto, desde la cibernética se establecen los requisitos para la conducción de dicho proceso y, por ende, del control de la asimilación cognoscitiva de los alumnos, como tarea más importante de la didáctica.

Talízina (1985) consideró que el profesor tiene dos funciones principales: ser fuente de información y dirigir el proceso de enseñanza y aprendizaje. Además expuso como exigencias de la cibernética a la dirección del proceso de enseñanza y aprendizaje, las siguientes: 1) definir el objeto de dirección (asimilación cognoscitiva del alumno-objeto de aprendizaje), 2) conocer los estados de transición del objeto de dirección a partir de objetivos a alcanzar, 3) realimentación-corrección (regulación).

El profesor, en su función dirigente, debe contribuir a desarrollar el trabajo consciente del grupo-alumno y, por ende, la co-dirección (bilateralidad) y la autodirección en el proceso de asimilación del alumno. Cuando se refiere que el profesor dirige el proceso de enseñanza y aprendizaje; no se entiende esta dirección como un acto autocrático y pasivo. Significa guiar, basado en un programa de estudio, el acto de aprendizaje del alumno de modo creativo, democrático, participativo y en estrecha relación entre sus actores. Reducir su función a facilitador, a mediador o a igual con el grupo-alumno, niega la relación existente entre los procesos de dirección y autodirección consciente. 
El estudio de la cibernética también permite aplicar en el ámbito didáctico las teorías de algoritmo y heurística, necesarias en la solución de problemas y en el desarrollo de la creatividad.

Didáctica-didáctica de la Geografía-sociología. Fuera de la sociedad no existe el ser humano, asumido como ser social. El principal camino de la educación de los individuos consiste, no en la inclusión de éste en los colectivos organizados, sino en la participación activa de éste en la formación de estos colectivos, en la organización de su vida y actividad, en las relaciones interpersonales en sus colectivos. La fuente sociológica también implica a la didáctica de la Geografía en el diseño de actividades docentes que contribuyan a la solución de los problemas sociales y, en particular, de la cotidianidad del alumno. Eso significa valorizar la función social de la ciencia que se enseña, es decir, comprender el valor de lo que se aprende y para qué se aprende.

Didáctica-didáctica de la Geografía-psicología. La conexión con la psicología está condicionada por el hecho que junto a los problemas de investigación diversos no coincidentes, tienen un objeto común; la formación de la psiquis humana. Cuanto más estrecho sea el contacto de la didáctica y de la didáctica de la Geografía con los últimos datos suministrados por la psicología tanto más fructífera será su labor. La penetración del método experimental en la psicología produjo toda una revolución en esta ciencia. El desarrollo del experimento psicológico se reflejó en la modelación del proceso de enseñanza y aprendizaje, así como en el perfeccionamiento de sus métodos. Destacar que la didáctica y, por ende, las didácticas especiales han admitido desde la década de 1950 a la psicología como fundamento esencial. Por esta razón, no son pocos los que entrelazan las teorías de aprendizaje de la psicología evolutiva, por su marcada influencia en el quehacer didáctico, al que trascienden por considerarse el aprendizaje del alumno como centro del proceso de enseñanza y aprendizaje.

Didáctica-didáctica de la Geografía-pedagogía. Se complementan mutuamente y existen relaciones esenciales entre estas ciencias pedagógicas, que tienen en su centro la educación de la personalidad. La didáctica y la didáctica de la Geografía utilizan aquellos conceptos que han sido formulados en los fundamentos de la pedagogía.

Tanto una como la otra, pedagogía y didáctica, son centro actualmente de un debate epistemológico, cuyo asunto versa sobre la cientificidad de ambas. Prevalecen, en ese sentido, diversas posiciones al respecto, definiéndolas como ciencia, como disciplina o como arte y, lo que es peor aún, hay quienes optan por su separación; que:

“(...) ha tenido peligrosas consecuencias para la práctica educativa, pues, a la larga, la pedagogía se ha debilitado como ciencia y la didáctica ha ocupado su lugar, por eso, ha recibido de una manera directa los fundamentos teóricos generales, y no, a través de la pedagogía. La didáctica, por otro lado, ha admitido a la psicología como su fundamento esencial. Por este motivo la clasificación de las didácticas al uso responde a las teorías psicológicas del aprendizaje”. (Chávez, Deler y Suárez, 2009, p.1) 
En ese sentido, las ciencias pedagógicas, son modeladas desde innumerables concepciones resultantes de una larga tradición histórica. Sustentadas, en su tiempo, por posiciones ideológicas, concepciones sobre la educación y el contexto socioeconómico de sus representantes. Estos modelos pedagógicos y didácticos se han reflejado en la modelación del proceso de enseñanza y aprendizaje geográfico en estrecha relación con los fundamentos epistemológicos de la Geografía. Así por ejemplo, Herrero (1995) y Alvarez (2014) establecen las relaciones siguientes (Cuadros 3 y 4):

\begin{tabular}{|c|c|}
\hline Tendencias geográficas & Tendencias educativas \\
\hline Ambientalista & Ecológica \\
\hline Regionalista & Ecológica \\
\hline Cuantitativa & Cognitiva \\
\hline Percepción & Conductista y cognitiva \\
\hline Radical & Ecológica e Interacción social \\
\hline Humanista & Ecológica \\
\hline
\end{tabular}

Cuadro 3. Relación entre las principales tendencias geográficas y educativas. Fuente: Herrero (1995).

\begin{tabular}{|c|c|c|}
\hline Enfoque geográfico & Base filosófica & Enfoque pedagógico y didáctico \\
\hline Determinismo geográfico & Positivismo & Pedagogía positivista \\
\hline Regional & $\begin{array}{l}\text { Historicismo con orientación ha- } \\
\text { cia el posibilismo geográfico }\end{array}$ & Pedagogía activa \\
\hline Cultural & Historicismo & Pedagogía social \\
\hline Teorético-cuantitativo & Neopositivismo & Pedagogía tecnológica \\
\hline Percepción & Neopositivismo & Conductismo \\
\hline Radical & Marxismo & Pedagogía marxista \\
\hline Humanística & Fenomenológica y existencial & Pedagogía trascendente \\
\hline Eco-geográfico & Ecléctica o electiva & Ecológica \\
\hline
\end{tabular}

Cuadro 4. Relación entre los enfoques geográficos y pedagógicos en el proceso de enseñanza y aprendizaje de la Geografía a partir de la base filosófica. Fuente: Alvarez (2014).

\section{DIDÁCTICA Y DIDÁCTICA DE LA GEOGRAFÍA: INVESTIGACIÓN E INNOVACIÓN}

La investigación didáctica es parte de la investigación pedagógica y se centra en la indagación y solución de los problemas relacionados con la eficiencia y eficacia del proceso de enseñanza y aprendizaje, así como con la dinámica de cualquiera de sus componentes culturales (problema-objetivo-contenido-método-medio-forma organizativa-evaluación) 
que median en la transmisión y asimilación cognoscitiva. En ésta se utilizan métodos de la investigación científica y, particularmente, de la investigación pedagógica.

En la investigación didáctica se tipifica, al igual que la investigación científica general, por su finalidad en investigación básica e investigación aplicada; y por su carácter de medida en cuantitativa, cualitativa o mixta.

La investigación didáctica, en la actualidad, se orienta con fuerza hacia la investigación aplicada. Esto, tal vez, influenciado por el modelo de gestión: investigación y desarrollo (I+D), aún predominante en muchas instituciones educacionales. Esta investigación aplicada se centra en los problemas de las didácticas especiales y, pocas veces, en los de la didáctica. Desatendiendo así, no solo la unidad teórico-metodológica al sobrevalorar las cuestiones metodológicas, sino que desestima los problemas teóricos que sustentan científicamente la aplicación de cualquier resultado en la práctica. Conduciendo esta situación a posiciones pragmáticas, que fracturan la necesaria relación práctica-teoríapráctica.

La innovación didáctica debe ser el concepto central que guíe la investigación didáctica. Se asume dicha innovación como los: "Cambios novedosos en uno de los componentes del proceso de enseñanza-aprendizaje que aportan nuevas formas de conocimiento, cambios originales en cualquiera de sus partes que en consecuencia traen aparejados la optimización en el logro de los objetivos, el perfeccionamiento de su dirección”. (Addine, 2004, p. 16)

En la didáctica y las didácticas especiales, desde la investigación, se debe continuar contribuyendo a la solución de problemas tales como: el aumento de la efectividad de la clase, el desarrollo de habilidades (capacidades y competencias) en la actividad de estudio y el trabajo independiente, así como la vinculación del enseñar y el aprender en la escuela y fuera de esta. Además de las líneas de investigación que se determinen en cada contexto y derivadas de la política educativa y demandas de la sociedad.

En la didáctica de la Geografía desde el sistema complejo de relaciones esenciales existentes, que ya se explicó, y que definen su unidad teórico-metodológica. La actividad investigativa y de innovación debe contribuir a la solución de problemas diversos y específicos de la enseñanza de la Geografía y de su necesario aprendizaje para la comprensión de las complejas relaciones naturaleza-naturaleza, sociedad-naturaleza y sociedadsociedad, en un lugar concreto del espacio geográfico y determinado escalarmente a nivel local, regional o global.

En las últimas tres décadas la investigación en didáctica de la Geografía se ha enriquecido en ámbitos de discusión (además de los nacionales) regionales e internacionales. Así por ejemplo, en nuestra región vale destacar las XVII ediciones, desde 1987 en Brasil, del Encuentro de Geógrafos de América Latina (EGAL), en los que no solo se intercambia experiencias de avanzada en investigación geográfica; sino que se discute sobre la enseñanza de la Geografía, como piedra angular de la futura formación de geógrafos; cues- 
tión que permite definir nuevas líneas de investigación, a partir de la situación problemática de la didáctica de la Geografía en cada país.

Se destaca, en el sentido anterior, la Comisión de Educación Geográfica de la Unión Geográfica Internacional (CEG-UGI) creada desde el año 1952.

La CEG-UGI, desde su creación, ha intentado aunar voluntades para la solución de los problemas didácticos de la Geografía. Vale destacar dos obras ejemplares y resultantes de la investigación didáctica mancomunada de prestigiosos investigadores: Método para la enseñanza de la Geografía (1966) y Nuevo Método para la Enseñanza de la Geografía (1989). Estas no solo contribuyeron a renovar la enseñanza de la Geografía, sino que se constituyeron como patrones de resultado de investigación en didáctica de la Geografía y expresión clara de su unidad teórico-metodológica.

Desde el pasado año 1992, la CEG-UGI y sus países miembros, han emitido una serie de declaraciones, en las que la investigación en didáctica de la Geografía ha ocupado un lugar central.

En la Declaración Internacional sobre Educación Geográfica (CEG-UGI, 1992) se definió la investigación, en educación geográfica, como la mejora de la enseñanza y aprendizaje de la Geografía en los niveles primario, secundaria, superior, formación profesional y educación de adultos; así como una contribución a la formulación de teorías generales que orientan la enseñanza y el aprendizaje. Para lograr tales fines, se requiere realizar investigaciones básicas como aplicadas.

En la Declaración Internacional sobre Educación Geográfica para la Diversidad Cultural (CEG-UGI, 2000) se insistió en la necesidad de contribuir, desde la investigación y enseñanza de la Geografía, a la comprensión de los ambientes culturales, sociales e industriales del mundo. Tema que cobra en la actualidad especial vigencia partir de la renovación de la geografía cultural.

En la Declaración de Lucerna sobre Educación Geográfica para el Desarrollo Sostenible (CEG-UGI, 2007) se reclamó la necesidad de la utilización de las tecnologías de la información y la comunicación en la investigación y la enseñanza de la Geografía para el logro del desarrollo sostenible.

En la Declaración Internacional sobre Investigación en Educación Geográfica (CEGUGI, 2016) se declararon cinco preguntas que se constituyen como línea de investigación para la didáctica de la Geografía en los próximos años: 1) ¿Qué Geografía necesitan conocer los estudiantes? ¿Cuáles son sus percepciones erróneas y concepciones previas? ¿Cómo podemos mejorar su conocimiento geográfico, su comprensión y sus habilidades geográficas? 2) ¿De qué manera tenemos constancia de que los estudiantes progresan en sus aprendizajes en Geografía? ¿Cómo estos progresos pueden ser medidos y evaluados de manera óptima? 3) ¿Cuáles son las características que hacen efectivos a los materiales y recursos de enseñanza y aprendizaje, en la mejora de la calidad de la educación geográfica? 4) ¿Qué métodos de enseñanza son eficientes y efectivos en la mejora de la calidad 
de la educación geográfica? 5) ¿Cómo puede mejorarse la formación de los profesores de Geografía para elevarla calidad de la docencia y el rendimiento en el aprendizaje de la Geografía escolar?

Las respuestas y soluciones, a estas preguntas, implican la necesaria integración de la didáctica de la Geografía con la didáctica y demás ciencias pedagógicas, que constituyen fuente y base de su unidad teórico-metodológica.

\section{CONCLUSIONES}

La reflexión teórica realizada permite llegar a las conclusiones siguientes:

- El análisis histórico de la didáctica y las didácticas especiales revela que éstas surgen integradas y diferenciándose posteriormente; pero con una unidad teórico-metodológica que se sustenta en las relaciones esenciales existentes. La didáctica especial aplica las teorías y metodologías generales de la didáctica, que utiliza los resultados de las didácticas especiales para su propio trabajo de generalización. Condición que se consolidará por la tendencia a la necesaria integración que se manifiesta entre las ciencias pedagógicas.

- En el análisis contextual, en Cuba, de la didáctica de la Geografía se verifica su origen como disciplina científico-académica independiente en la década de los '60. Mostrando en su evolución la necesaria interdependencia con la didáctica y las demás ciencias pedagógicas.

- La unidad teórico-metodológica de la didáctica y la didáctica de la Geografía construye sus bases desde fundamentos filosóficos, cibernéticos, sociológicos, psicológicos y pedagógicos; los que constituyen fuente en la modelación del proceso de enseñanza y aprendizaje geográfico.

- La investigación didáctica, en la actualidad, se orienta con fuerza hacia la investigación aplicada en las didácticas especiales; cuestión que atenta contra la solución de los problemas teóricos que sustentan científicamente la aplicación de cualquier resultado en la práctica. Asumiéndose así, posiciones pragmáticas que fracturan la necesaria relación práctica-teoría-práctica y, por ende, la unidad teórico-metodológica. Esta situación se refleja en la investigación en didáctica de la Geografía; lo que implica un cambio en el quehacer científico nucleado desde la necesaria innovación didáctica y relación entre los fundamentos filosóficos, cibernéticos, sociológicos, psicológicos, pedagógicos y didácticos. 


\section{REFERENCIAS}

Addine, F. (2004). ¡Didáctica! ¿Qué Didáctica? En F. Addine, Didáctica: teoría y práctica (págs. 1-20). La Habana: Editorial Pueblo y Educación.

Alvarez, P. (2014). Enfoque cooperativo-transformativo del poceso de enseñanza-aprendizaje de la Geografía en la formación del profesor de esta disciplina. Tesis doctoral. La Habana: Universidad de Ciencias Pedagógicas Enrique José Varona.

Alvarez, P., Pérez, C. E. y Recio, P. P. (2015). La formación del profesor de Geografía en Cuba ante la pluralidad de enfoques epistemológicos de la ciencia geográfica. Retos y perspectivas. Varona (61), 58-70.

Barraqué, G. (1978, 1991). Metodología de la enseñanza de la Geografía (Primera y segunda Ed.). La Habana: Editorial Pueblo y Educación.

Brouillette, B. et al. (1966). Método para la enseñanza de la geografía (Segunda ed.). Barcelona: Editorial Teide S. A.

CEG-UGI. (1992). Declaración Internacional de Educación Geográfica. Washington D. C.: Comisión de Educación Geográfica-Unión Geográfica Internacional. Recuperado el 22 de Abril de 2019, de [Enlace]

CEG-UGI. (2000). Declaración Internacional de Educación Geográfica.Seúl: Comisión de Educación Geográfica-Unión Geográfica Internacional. Recuperado el 22 de Abril de 2019, de [Enlace]

CEG-UGI. (2007). Declaración de Lucerna sobre Educación Geográfica para el Desarrollo Sostenible.Lucerna: Comisión de Educación Geográfica-Unión Geográfica Internacional. Recuperado el 22 de Abril de 2019, de [Enlace]

CEG-UGI. (2016). Declaración Internacional sobre Investigación en Educación Geográfica. Moscú: Comisión de Educación Geográfica-Unión Geográfica Internacional. Recuperado el 22 de Abril de 2019, de [Enlace]

Chávez, J. A., Deler, G. y Suárez, A. (2009). Principales corrientes y tendencias a inicios del siglo XIX de la pedagogía y la didáctica. La Habana: Editorial Pueblo y Educación.

Comenio, J. A. (1983). Didáctica Magna (Tomado de la edición en español de la Editorial Reus S. A., Madrid, 1922). La Habana: Editorial Pueblo y Educación.

Danilov, M. A. y Skatkin, M. N. (1978). Didáctica de la escuela media (Primera edición cubana ed.). La Habana: Editorial Pueblo y educación.

Darinsky, A. V. (1975). Metodología de la enseñanza de la Geografía. Moscú: Editorial Instrucción.

Graves, N. J., et al. (1989). Nuevo método para la enseñanza de la geografía (Primera ed.). Barcelona: Editorial Teide S. A. 
Hernández, P. A., et al. (1999). Enrutando la Geografía Escolar. La Habana: Editorial Academia.

Hernández, P. A., et al. (2002). El perfeccionamiento del currículo de Geografía en la escuela cubana actual. En C. E. Pérez, et al. Selección de temas de Didáctica de la Geografía (págs. 32-42). La Habana: Editorial Pueblo y Educación.

Herrero, C. (1995). Geografía y Educación. Madrid: Huerga y Fierro editores.

Klingberg, L. (1978). Introducción a la Didáctica General. La Habana: Editorial Pueblo y Educación.

Ministerio de Educación, Cuba. (1975). Didáctica de la Geografía. Programa-Guía. Cuarto año. Carrera profesoral de Geografía. La Habana: Editorial Pueblo y Educación.

Pancheshnikova, L. M., et al. (1983). Metodología de la enseñanza de la Geografía. Moscú: Editorial Proveschenie.

Pérez, C. E. y Alvarez, P. (2018). Didáctica de la Geografía. La Habana: Editorial Universitaria Félix Varela.

Pérez, C. E. y Recio, P. P. (2002). ¿Didáctica de la Geografía o Metodología de la enseñanza de la Geografía? En C. E. Pérez, et al. Selección de temas de Didáctica de la Geografía (págs. 16-20). La Habana: Editorial Pueblo y educación.

Pérez, C. E., et al. (2002). Selcción de temas de Didáctica de la Geografía. La Habana: Editorial Pueblo y Educación.

Rosental, M. y Iudin, P. (1973). Diccionario filosófico. Argentina: Ediciones Universo.

Santarelli, S. y Campos, M. (2008). Corrientes epistemológicas actuales en geografía. Experiencias en enseñanza e investigación. Seminario Participativo. Institucional del Instituto Superior de Formación Docente No 79. Buenos Aires.

Studensov, N. N. (1966). La lección de Geografía en la escuela. La Habana: Editorial Nacional de Cuba.

Talízina, N. F. (1985). Conferencias sobre los fundamentos de la enseñanza en la educación superior. La Habana: Deparatmento de Estudios para el Perfeccionamiento de la Educación Superior.

Tomaschewky, K. (1974). Didáctica (Tercera ed.). México D. F.: Editorial Grijalbo. 